\title{
POLARIZATION SWINGS REVEAL MAGNETIC ENERGY DISSIPATION IN BLAZARS
}

\author{
Haocheng Zhang ${ }^{1,2}$, Xuhui Chen ${ }^{3,4}$, Markus Böttcher ${ }^{1,5}$, Fan Guo ${ }^{2}$, and Hui $\mathrm{LI}^{2}$ \\ ${ }^{1}$ Astrophysical Institute, Department of Physics and Astronomy, Ohio University, Athens, OH 45701, USA; hz193909@ohio.edu \\ 2 Theoretical Division, Los Alamos National Laboratory, Los Alamos, NM 87545, USA \\ ${ }^{3}$ Institute of Physics and Astronomy, University of Potsdam, D-14476 Potsdam-Golm, Germany \\ ${ }^{4}$ DESY, Platanenallee 6, D-15738 Zeuthen, Germany \\ ${ }^{5}$ Centre for Space Research, North-West University, Potchefstroom, 2520, South Africa \\ Received 2014 November 1; accepted 2015 February 26; published 2015 April 30
}

\begin{abstract}
The polarization signatures of blazar emissions are known to be highly variable. In addition to small fluctuations of the polarization angle around a mean value, large $\left(\gtrsim 180^{\circ}\right)$ polarization angle swings are sometimes observed. We suggest that such phenomena can be interpreted as arising from light travel time effects within an underlying axisymmetric emission region. We present the first simultaneous fitting of the multi-wavelength spectrum, variability, and time-dependent polarization features of a correlated optical and gamma-ray flaring event of the prominent blazar 3C279, which was accompanied by a drastic change in its polarization signatures. This unprecedented combination of spectral, variability, and polarization information in a coherent physical model allows us to place stringent constraints on the particle acceleration and magnetic field topology in the relativistic jet of a blazar, strongly favoring a scenario in which magnetic energy dissipation is the primary driver of the flare event.
\end{abstract}

Key words: galaxies: active - galaxies: jets - gamma-rays: galaxies - radiation mechanisms: nonthermal relativistic processes

\section{INTRODUCTION}

Blazars are the most violent active galactic nuclei. They are known to emit nonthermal-dominated radiation from radio frequencies to $\gamma$-rays with strong variability across the entire spectrum (e.g., Aharonian et al. 2007). Such emission likely originates from a relativistic jet that is directed close to our line of sight (LOS). The blazar spectral energy distribution (SED) has two components. It is generally acknowledged that the nonthermal radio through optical-UV emission is synchrotron radiation from ultrarelativistic electrons. In leptonic models, the high-energy component, from X-rays to $\gamma$-rays, is usually interpreted as resulting from inverse Compton scattering by the same nonthermal electron population of soft seed photons. Seed photons can either come from the synchrotron component itself (synchrotron-self Compton, SSC; e.g., Marscher \& Gear 1985; Maraschi et al. 1992), or from external photon fields originaiting in the broad line region and/or a dusty torus (external Compton, EC; e.g., Dermer et al. 1992; Sikora et al. 1994). However, a hadronic origin of the high-energy component cannot be ruled out (e.g., Mannheim \& Biermann 1992; Mücke \& Protheroe 2001; Böttcher et al. 2013). Recently, Zhang \& Böttcher (2013) demonstrated that highenergy polarization signatures can serve as a powerful diagnostic between the leptonic and hadronic processes.

Despite intensive observational and theoretical efforts, the particle acceleration mechanism, the energy source for the flaring activity, and the inner jet physical conditions, such as the magnetic field topology, are not well understood. On the observational side, the synchrotron component is known to be polarized with polarization degrees ranging from a few to tens of percent, in agreement with a synchrotron origin in a partially ordered magnetic field. Several authors (e.g., Lyutikov et al. 2005; Pushkarev et al. 2005) have shown that the observed polarization degree and angle may reveal a helical magnetic field structure. Both the polarization degree and angle are known to be highly variable. Large $\left(\geqslant 180^{\circ}\right)$ polarization angle swings have been frequently observed, and some recent observations (Marscher et al. 2008, 2010; Abdo et al. 2010a) have shown that they are sometimes accompanied by simultaneous optical and $\gamma$-ray flaring activities. When the polarization angle is not rotating, small fluctuations of the polarization signatures around a relatively stable mean value are often observed (e.g., Aleksić et al. 2014; Morozova et al. 2014). Time-symmetric polarization profiles are observed in both cases; in particular, a complete $\sim 180^{\circ}$ polarization angle swing is often accompanied by a drop of the polarization degree to nearly zero, followed by a recovery back to its value before the polarization angle (PA) rotation (see the above references). On the theoretical side, the general formalism for synchrotron polarization is well understood (e.g., Westfold 1959). Nevertheless, the spectrum, variability, and polarization signatures have never been combined into one coherent physical model to understand the inner jet physics. Models for synchrotron polarization necessarily take into account the magnetic field topology and polarization-dependent synchrotron emissivity, but often apply simple, time-independent, power-law electron populations, and usually ignore the generation of high-energy emission (e.g., Lyutikov et al. 2005). Thus, they cannot produce the full broadband SED and variability. On the other hand, models presenting detailed calculations of broadband emission and variability typically assume a chaotic magnetic field and ignore any polarizationdependent emissivity and polarization information. Additionally, previous emission models hardly provide any constraints on the particle acceleration mechanisms and the energy source. In a recent paper, Zhang et al. (2014; hereafter Paper I) have presented a general parameter study as the first step in combining the full SED, variability, and polarization signatures into a single physical model. That paper has demonstrated that PA swings, correlated with high-energy flaring activity, only require a dominant helical magnetic field structure in a straight 
jet, but no non-axisymmetric jet features, such as bends or helical flow streamlines.

In this paper, we will investigate additional geometric effects, namely, the shape of the emission region and the magnetic field topology, the synchrotron polarization signatures, and multi-wavelength variability. We find that the apparently time-symmetric polarization profiles can be obtained by an axisymmetric emission region along with full consideration of the light-travel-time effects (LTTEs). We will then apply the results obtained here and in Paper I to present the first simultaneous fitting of snapshot broadband SEDs, light curves in various frequency bands, and time-dependent polarization signatures from a correlated optical and $\gamma$-ray flaring event of the prominent blazar 3C279, which was accompanied by a drastic change in its polarization features. This unprecedented fitting combination in one coherent model allows us to place stringent constraints on the particle acceleration and its energy source, as well as the magnetic field structure and its variation, in the inner jet of the blazar. Our results strongly favor a scenario in which magnetic energy dissipation is the primary driver of the flare event. We will describe our model setup in Section 2, present a general study of the geometric effects in Section 3, fit the 3C279 flare event in Section 4, and discuss the results in Section 5.

\section{MODEL SETUP}

Our setup is designed to highlight the combined geometric and LTTEs with the simplest physical assumptions. The underlying model considered here assumes a cylindrical emission region which travels on a straight trajectory defined by the jet boundary and encounters a flat stationary disturbance. The entire region is pervaded by a predominantly helical magnetic field with a possible additional turbulent component. The origin of the disturbance could be a shock, which will convert bulk kinetic energy into random motion of relativistic particles to generate a flare, but it may also may also be associated with other mechanisms such as magnetic reconnection, which will dissipate magnetic energy to accelerate particles (Guo et al. 2014, see Section 4). In the comoving frame of the emission region, the disturbance will travel through the emission region and temporarily change the plasma conditions at its location, primarily the magnetic field structure and the nonthermal particle distribution through a modification of the acceleration timescale, and hence generate a flare. As the disturbance leaves a given point in the emission region, the plasma conditions will recover back to the initial state. Most importantly, due to the LTTEs, the observer will receive signatures of flaring activities induced by the disturbance from points at different longitudinal distances along the jet at the same time. Due to the axisymmetry of the emission region, this leads to time-symmetric polarization profiles (see Sections 3 and 4).

The above model is simulated by the combination of the two-dimensional Monte-Carlo/Fokker-Planck radiation transfer (MCFP) code developed by Chen et al. $(2012,2014)$ and the $3 \mathrm{D}$ multi-zone synchrotron polarization ray-tracing code developed by Zhang et al. (2014). Figure 1 shows the model and code setup. The MCFP code considers a cylindrical emission region, assumes axisymmetry for all physical properties, and divides the region evenly into multiple zones in the radial and longitudinal directions. The electron distribution in each zone evolves in time according to a locally isotropic
Fokker-Planck equation. The disturbance is also a cylindrical region, which will instantaneously modify the physical conditions of the zones at its current location, hence modifying the Fokker-Planck evolution. When it moves out of a zone, the physical conditions will instantaneously be restored to the initial state. Here, the physical meaning of an "instantaneous" change is that its timescale is less than one time step of the simulation. The code will then calculate the time-dependent emissivities based on the particle distribution at each time step, and apply a Monte-Carlo method to trace the photons and handle the Compton scattering. Therefore, all LTTEs are naturally included. All calculations are performed in the comoving frame of the emission region, and the resulting fluxes are properly transformed to the observer's frame at the end of the simulation.

The 3DPol code is focused on the synchrotron polarization signatures. It uses the same geometry and physical conditions, and the particle distributions as calculated by the MCFP code. However, since the polarization requires a 3D description, the emission region is further divided evenly into multiple zones in the $\phi$ direction, but the axisymmetry of all parameters is still kept. Since the environment of the emission region is generally considered optically thin, and our treatment of the synchrotron polarization focuses on the high-frequency radio through optical/UV range, synchrotron-self absorption and Faraday rotation effects are neglected in this study. As in the MCFP code, all calculations are performed in the comoving frame. In each zone, we project the magnetic field onto the plane of sky in the comoving frame and use the time-dependent electron population generated by the MCFP code to evaluate the polarization degree (PD) at various frequencies. Since the net electric vector is perpendicular to the projected magnetic field direction, the electric vector position angle, also known as the polarization angle (PA), is easily obtained. In this way, we obtain the Stokes parameters (without normalization) at various frequencies at every time step in each zone via

$$
(I, Q, U)_{\nu}=L_{\nu} *\left(1, \Pi_{\nu} \cos 2 \theta_{E}, \Pi_{\nu} \sin 2 \theta_{E}\right)
$$

where $L_{\nu}$ and $\Pi_{\nu}$ are the spectral luminosity and the PD at frequency $\nu$, respectively, and $\theta_{E}$ is the electric vector position angle for that zone. The code then employs ray-tracing to calculate the relative time delay to the observer for each zone in order to fully account for the LTTEs. Since the emissions from different zones are incoherent, the total Stokes parameters can be obtained through the linear addition of the Stokes parameters from each zone for emissions reaching the observer at the same time. Finally, as in the MCFP code, all of the emissions are properly Lorentz transformed (boosted) to obtain the luminosity and polarization information in the observer's frame.

Due to the relativistic aberration, even though we are observing blazars nearly along the jet in the observer's frame (typically, $\theta_{\mathrm{obs}}^{*} \sim 1 / \Gamma$, where $\Gamma$ is the bulk Lorentz factor of the outflow along the jet), the angle $\theta_{\text {obs }}$ between LOS and the jet axis in the comoving frame is likely much larger. Specifically, if $\theta_{\mathrm{obs}}^{*}=1 / \Gamma$, then $\theta_{\mathrm{obs}}=90^{\circ}$. Paper I has shown that the polarization signatures are only weakly dependent on the exact value of $\theta_{\text {obs }}$, thus in all of the following simulations, we choose $\theta_{\mathrm{obs}}=90^{\circ}$ in the comoving frame, and hence the Doppler factor $\delta \equiv\left(\Gamma\left[1-\beta_{\Gamma} \cos \theta_{\mathrm{obs}}^{*}\right]\right)^{-1}=\Gamma$. 

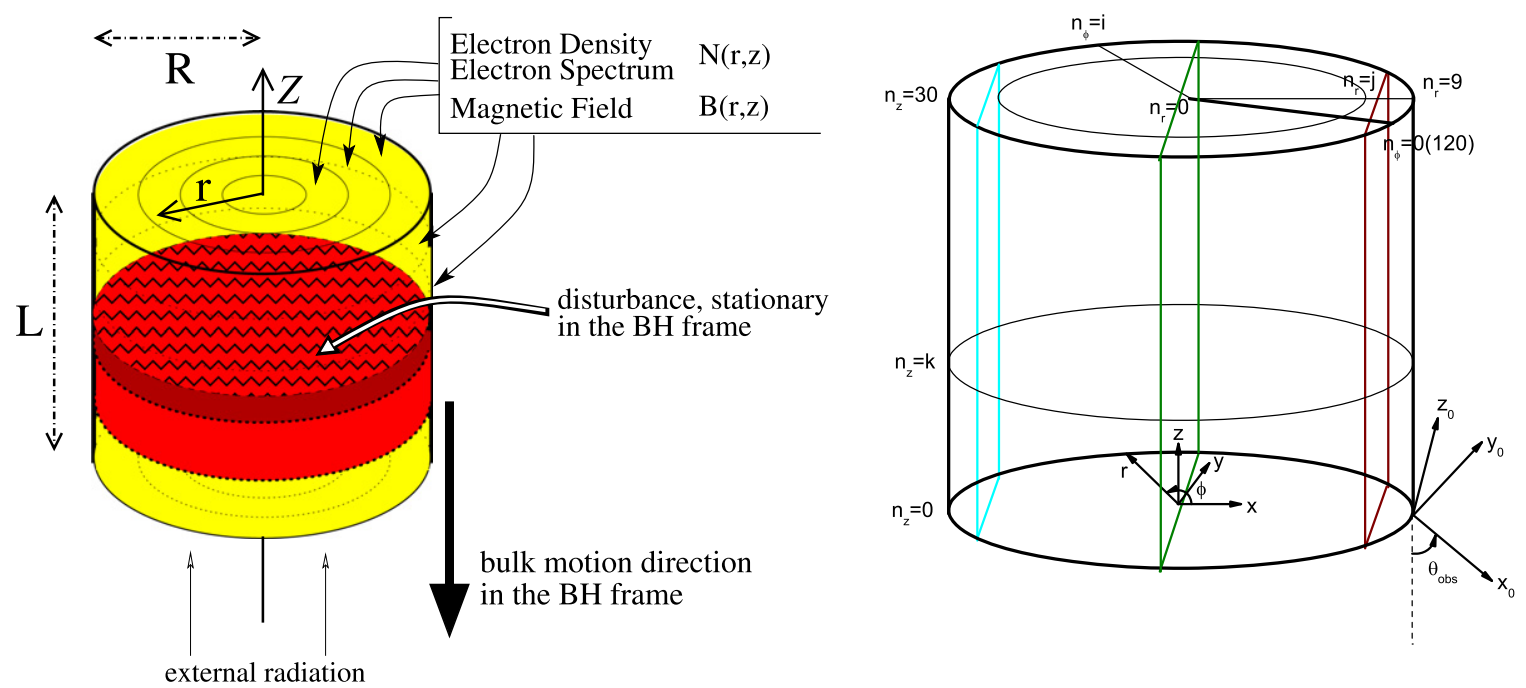

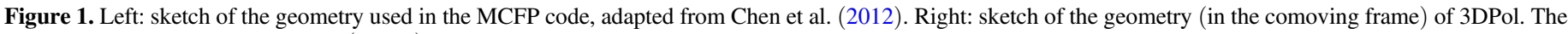

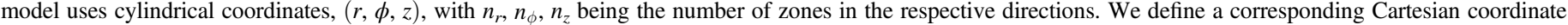

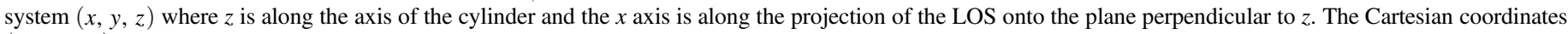

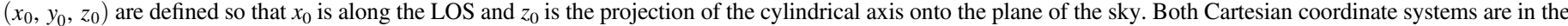

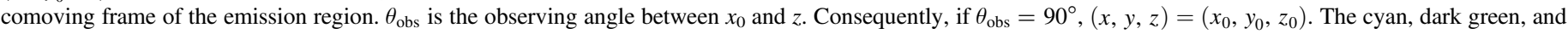
maroon regions represent the far side (left), middle, and near side (right) zones, respectively.

\section{GEOMETRIC EFFECTS}

In this section, we use the quasar PKS 1510-089 as an example to investigate a number of generic flaring scenarios as case studies. PKS 1510-089 is a flat-spectrum radio quasar (FSRQ). Its flaring activity in 2009 March was well covered by multi-band monitoring in the infrared, optical, X-rays, and $\gamma$ rays (Abdo et al. 2010b; Marscher et al. 2010; D'Ammando et al. 2011). Chen et al. (2012) presented model fits to snapshot SEDs and light curves, in which they found that the most favorable scenario is an EC model with the external radiation field originating from a dusty torus.

The purpose of this section is to study geometric effects on the polarization signatures, in particular, the PA variability. The geometries are chosen in such a way that the first two cases correspond to $180^{\circ} \mathrm{PA}$ swings, while the other two cases are intended to study small-scale PA fluctuations. In order to facilitate a direct comparison with Paper I, we choose the same quiescent state parameters, similar to those used in Chen et al. (2012). In addition, we maintain the assumption that the disturbance is due to a shock. However, the shock parameters and the geometry may vary. Also, for the purpose of these generic case studies, we do not introduce any turbulent magnetic field contribution. Since both the MCFP and 3DPol codes are time-dependent, we allow for an initial period for the electrons and the photons to reach equilibrium before we introduce the parameter disturbance produced by the shock. In all of our results, the plots illustrate the results after this equilibrium has been reached. As the flaring activity exhibits different characteristics in duration and strength for different cases, we define similar phases in the flare development for the purpose of a direct comparison. These phases correspond approximately to the pre-flare, early flare, flare peak, late flare, and post-flare states.

We define the PA in our simulations as follows. PA $=0$ corresponds to the electric field vector being parallel to the projection of the cylindrical axis on the plane of sky. An increasing PA corresponds to counter-clockwise rotation with respect to the LOS and to $180^{\circ}$ when it is anti-parallel to the projected cylindrical axis (which is equivalent to 0 due to the $180^{\circ}$ ambiguity). Based on the above definition, the Stokes parameters normalized by luminosity for one zone with its projected magnetic field directed in the range of 0 to $45^{\circ}$ is in the form of $(1,-|q|,|u|)$, in the range $45^{\circ}$ to $90^{\circ}$ it is $(1,|q|,|u|)$, for $-45^{\circ}$ to 0 it is $(1,-|q|,-|u|)$, and for $-90^{\circ}$ to $45^{\circ}$ it is $(1,|q|,-|u|)$. This convention will be frequently used in the following text. All of our results are shown in the observer's frame.

Table 1 lists some key parameters. The emission region is a cylinder of length $Z$ and radius $R$, traveling at a bulk Lorentz factor of $\Gamma$ in the observer's frame, when it encounters a flat, uniform, stationary disturbance of length $L$ and radius $A$. The Fokker-Planck equation takes into account the acceleration timescale $t_{\text {acc }}$ and escape timescale $t_{\mathrm{esc}}$, both in units of $Z / c$; however, the total number of electrons is conserved, and hence the escaping electrons will be balanced by thermal electrons picked up from the background. In the quiescent state, the emission region is pervaded by a helical magnetic field $B_{H}$ oriented at a pitch angle $\theta_{B}$, and possibly a turbulent contribution where the total magnetic field strength is $B$. However, in this geometric effect study, we do not introduce any turbulence, therefore $B_{H}=B$. Initially, a power-law distribution of nonthermal electrons with a power-index $p$ and minimum and maximum energies $\gamma_{\min }$ and $\gamma_{\max }$, respectively, with density $n_{e}$, will have evolved to an equilibrium according to the Fokker-Planck equation in the emission region before the interaction with the disturbance. The disturbance will change the layers in the emission region at its location into an active state. In cases 1,2 , and 4 , the disturbance, which is in the form of a shock, will compress the local magnetic field so that the magnetic field strength will be amplified by a factor of $B^{s} / B$, and the pitch angle will change to $\theta_{B}^{s}$. In case 3 , the shock will instead shorten the acceleration timescale by a factor of $t_{\mathrm{acc}}^{s} / t_{\mathrm{acc}}$, so as to increase the acceleration efficiency. 
Table 1

Summary of Model Parameters

\begin{tabular}{|c|c|c|c|c|}
\hline Parameters & General Properties & & & \\
\hline Total (helical) magnetic field $B(G)$ & 0.2 & & & \\
\hline Initial electron density $n_{e}\left(10^{2} \mathrm{~cm}^{-3}\right)$ & 7.37 & & & \\
\hline Initial electron minimum energy $\gamma_{\min }$ & 50 & & & \\
\hline Initial electron maximum energy $\gamma_{\max }$ & 20000 & & & \\
\hline Initial electron spectral index $p$ & 3.2 & & & \\
\hline Orientation of LOS $\theta_{\mathrm{obs}}\left(^{\circ}\right)$ & 90 & & & \\
\hline Parameters & Case 1 & Case 2 & Case 3 & Case 4 \\
\hline Helical pitch angle $\theta_{B}\left({ }^{\circ}\right)$ & 45 & 45 & 65 & 45 \\
\hline Length of the emission region $Z\left(10^{16} \mathrm{~cm}\right)$ & 8.0 & 18.0 & 18.0 & 8.0 \\
\hline$\theta_{B}^{s}\left({ }^{\circ}\right)$ & 75 & 75 & $\cdots$ & 75 \\
\hline$t_{\mathrm{acc}}^{s} / t_{\mathrm{acc}}$ & $\cdots$ & $\cdots$ & $1 / 2.5$ & $\cdots$ \\
\hline
\end{tabular}

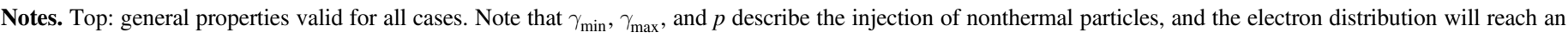

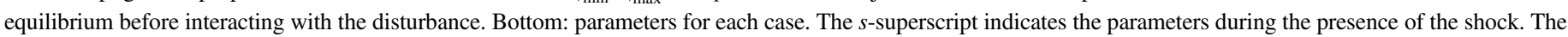

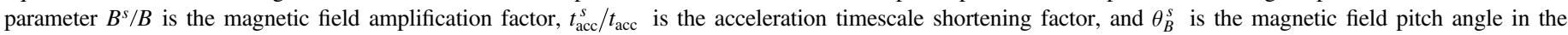
shocked region.

\subsection{B-field Compression, $Z<2 R$}

In this scenario, the shock will instantaneously increase the toroidal magnetic field component at its location, probably by compression, so that it will increase the total magnetic field strength and change its orientation in the affected zones. The new magnetic field will be retained until the shock moves out of that zone, when it instantaneously reverts back to its original (quiescent) strength and orientation, which can be attributed to dissipation. Paper I considered a very extreme case where the shock increases the toroidal component by a factor of 10 , so that the total magnetic field is increased by approximately a factor of 7 , and $\theta_{B}$ increases from initially $45^{\circ}$ to approximately $84^{\circ}$. Such a strong alteration in the magnetic field results in a drastic change in the polarization signatures; however, it will require a huge amount of energy conversion within small time windows when the disturbance is turned on and off, which is unlikely to happen in practice. Here, we investigate a case with a moderate change in the magnetic field, where $\theta_{B}$ of the total magnetic field increases from $45^{\circ}$ to $75^{\circ}$. To mimic a spherical volume for the emission region, we choose $R: Z=1: \frac{4}{3}$, so that $Z<2 R$, and the disturbance will occupy the entire layer, i.e., $A=R$ (hereafter, Geometry I).

Since the total magnetic field does not increase much, in Figure 2 (upper left) we observe that the synchrotron flux exhibits only a small flare. It is also obvious that the PD is photon-energy dependent (Figure 2 lower left) since PKS 1510-089 has a visible external thermal component emerging at optical-UV frequencies, which diminishes the observed polarization percentage, especially in the UV. The dotted lines in Figure 2 (lower left) show the intrinsic PD without thermal contamination. The spectral curvature of the realistic electron spectrum from the detailed simulation based on the Fokker-Planck equation leads to an additional frequency dependence of the PD. The features above $\sim 100 \mathrm{eV}$ are due to the electron spectral cutoff. For the time dependence of the polarization signatures, the PD gradually decreases to nearly zero at the flare peak, then recovers back to its initial state at the end of the flare. Moreover, the PA shows a continuous $180^{\circ}$ swing, except for the radio to millimeter polarization, which is due to the extremely small flare amplitude at low frequencies. As elaborated below and in Paper I, such polarization variability features can be explained as the combined effect of electron evolution and LTTEs.

Initially, the directions of the projected magnetic field will cover the range from $-45^{\circ}$ to $45^{\circ}$. Due to the axisymmetry of the initial conditions, the initial $|u|$ component will be balanced out, and hence the initial Stokes vector is $(1,-|q|, 0)$, leaving the PA at $270^{\circ}$ (equivalent to $90^{\circ}$ ). When the shock moves into the emission region, it will increase the toroidal component of the magnetic field at its location, resulting in stronger emission and enhanced synchrotron cooling (Figure 3 left). Additionally, during the presence of the disturbance, the active region has $\theta_{B}=75^{\circ}$. The combined effect is that the active region will have a larger contribution to the polarization, with the Stokes parameters generally in the form of $(1,|q|,|u|)$ or $(1,|q|,-|u|)$. Due to LTTEs, although the shock is flat in the comoving frame, the active region at equal photon-arrival times will be distorted, as shown in Figures 4(a) and (b). In the early flare phase, only the region facing the observer (red in Figure 4) contributes to the flare, where the initial Stokes parameters in the form of $(1,-|q|,|u|)$ will be replaced by the active state $(1,|q|,|u|)$. Therefore, the initial $P A=270^{\circ}$ will gradually move to $225^{\circ}$ and the PD will decrease at the same time. When the flare gradually rises to its peak, emission from the far side of the cylinder can be seen which possess a negative $u$ component. This will diminish the positive $u$ from the right side of the cylinder; meanwhile, the initial negative $q$ 

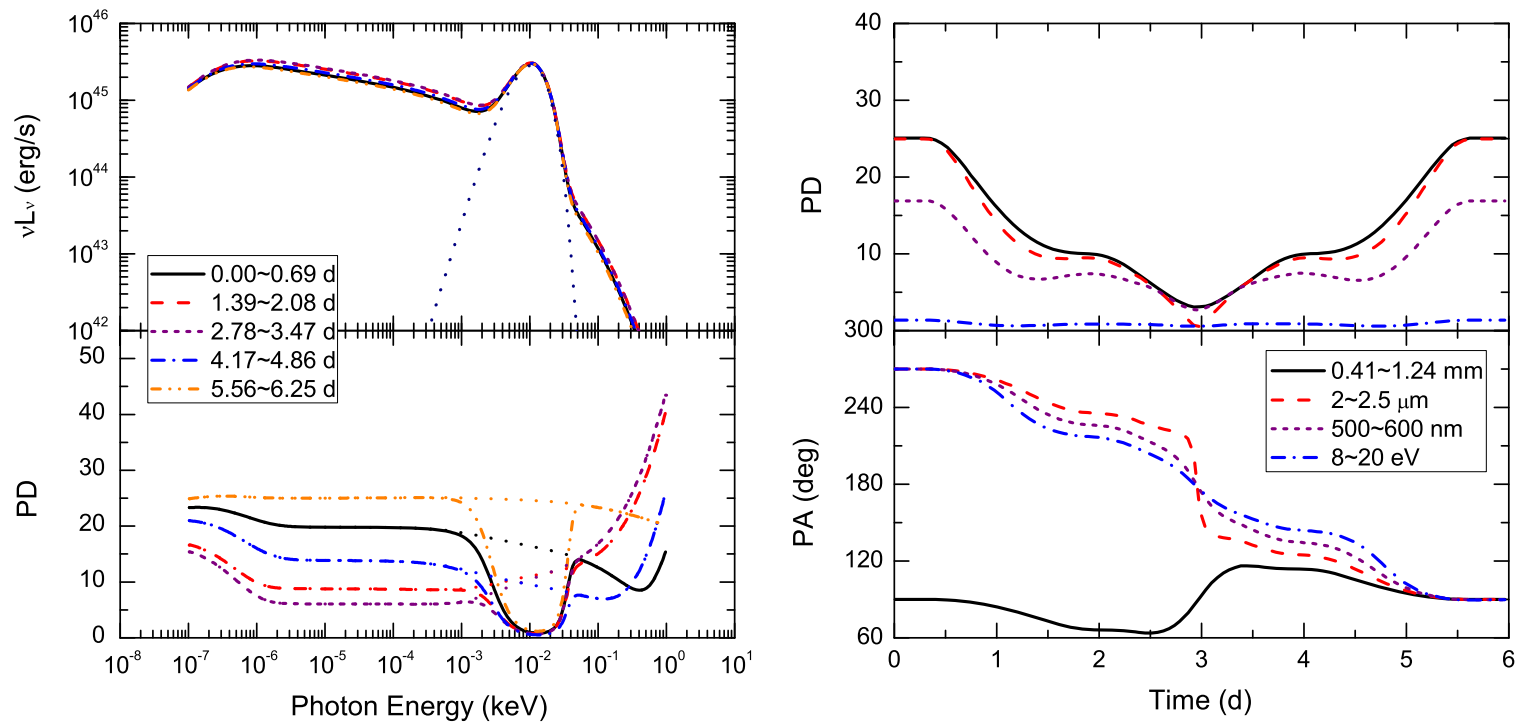

Figure 2. Case 1: a moderate change of magnetic field in Geometry I. Upper left: snapshot synchrotron SEDs, including the external photon field. SEDs are chosen at approximately the pre-flare (black solid), early flare (red dashed), peak (purple short dashed), late flare (blue dash dot), and back to equilibrium at the post-flare (orange dash dot dot) states, with the dotted line for the external photon field contribution. All SEDs are chosen with the same time bin size. Lower left: PD vs. photon energy for the same time bins as the SEDs in the top panel, with the external photon contamination considered, where the dotted lines represent the PD without the contamination. Upper right: PD vs. time with external contamination, at radio (black solid), infrared (red dashed), optical V band (purple short dashed), and UV (blue dash dot). Lower right: PA vs. time for the energy bands as in the top panel.
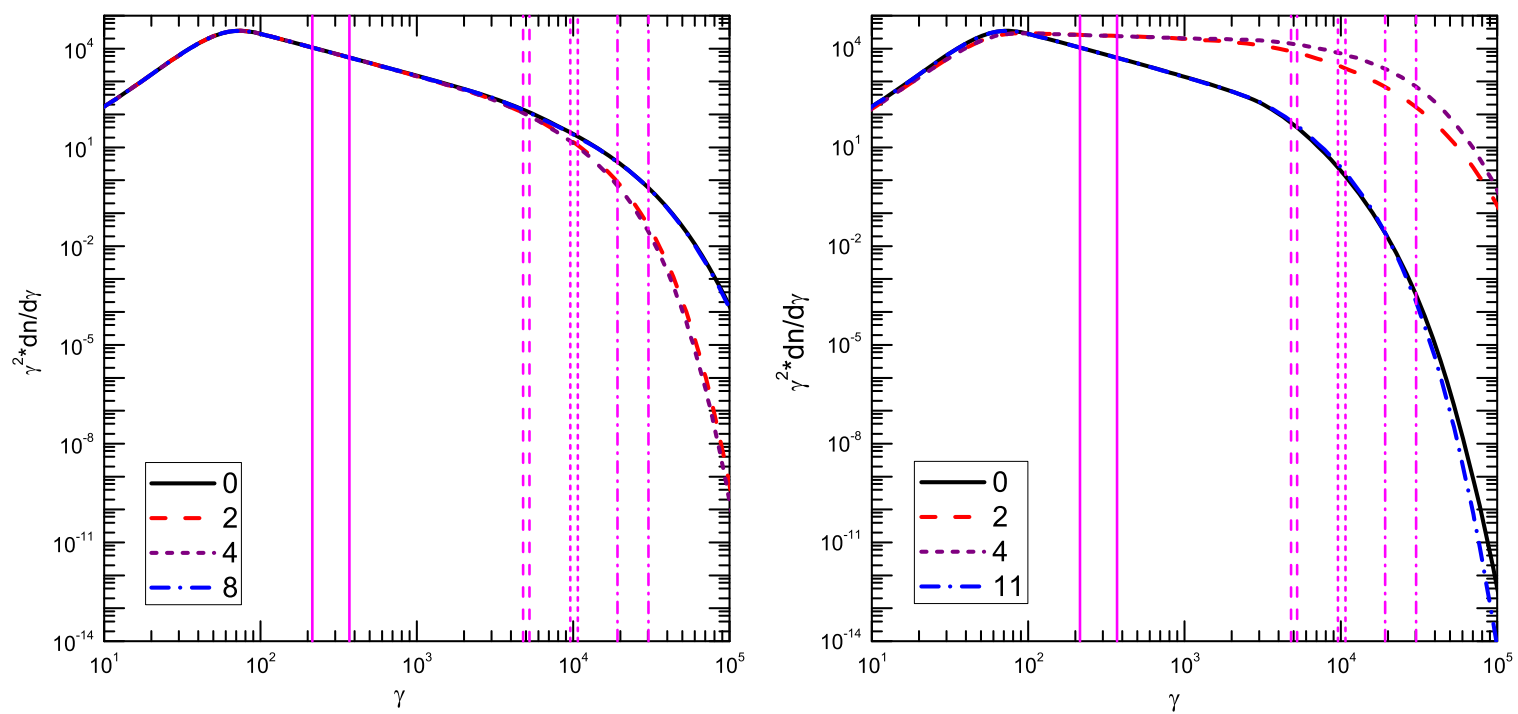

Figure 3. Electron spectra at different time steps for a moderate change in the magnetic field (left) and a shortening of the acceleration timescale (right). 0: pre-flare equilibrium; 2: central shock position; 4: shock just leaves the emission region; 8(11): post-shock equilibrium. The regions between the magenta vertical lines represent the electron energies that correspond to the photon energies we choose in the polarization vs. time plots: solid corresponds to radio, dashed to infrared, short dashed to optical, and dashed-dotted to UV.

has mostly been canceled out. Consequently, the PD will continue to drop and the PA will rotate from $225^{\circ}$ to $180^{\circ}$. When the flare reaches its maximum (green in Figure $4(\mathrm{~b})$ ), for a short period the shock-enhanced fluxes from both sides of the cylinder will be comparable. As a result, the $u$ component will be canceled out, leaving a positive $q$ in the active region and a negative $q$ in the quiescent region. If the shock is adequately strong, then the polarization contributed by the active region will be higher, leading to a positive $q$ for this short period. Therefore, the PD drops to nearly zero for all bands while the PA reaches $180^{\circ}$ for the infrared, optical, and UV. However, the PA rotates back to the initial $90^{\circ}$ for the radio, as its flare amplitude is not strong enough to dominate over the quiescent emission. After the peak, the flaring region moves to the far side, so that the polarization signatures gradually revert back to the initial state in time-symmetric patterns.

\subsection{B-field Compression, $Z>2 R$}

In order to isolate the dependence of the polarization signatures on the geometry of the emission region, we studied a scenario in which we kept all of the parameters exactly the same as in the previous case, except for the size of the emission region. We choose an emission region with the same volume, but now $R: Z=\frac{2}{3}: 3$ (hereafter, Geometry II). The general electron spectrum is similar to the previous case, except for minor differences due to the electron cooling rate, escape rate, 
a)

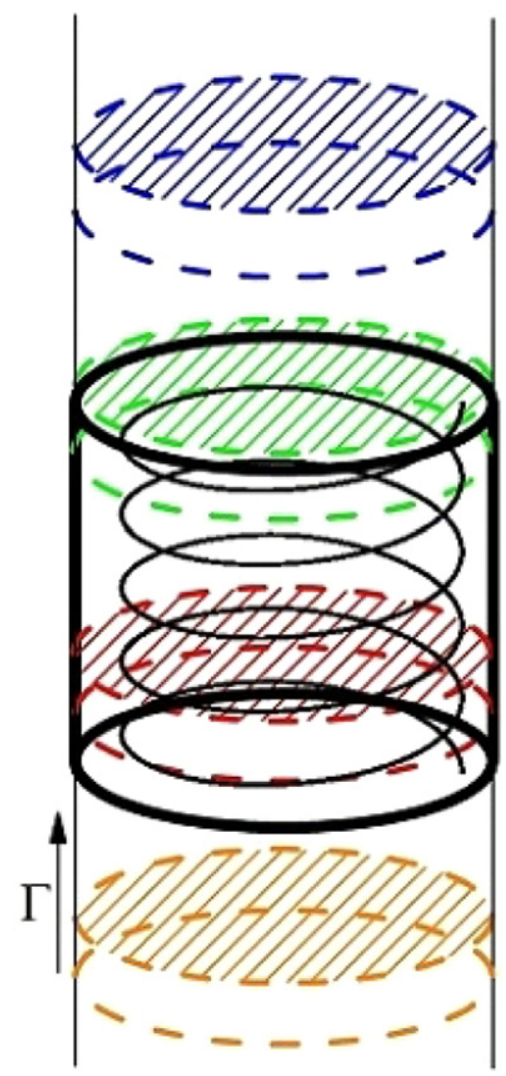

b)

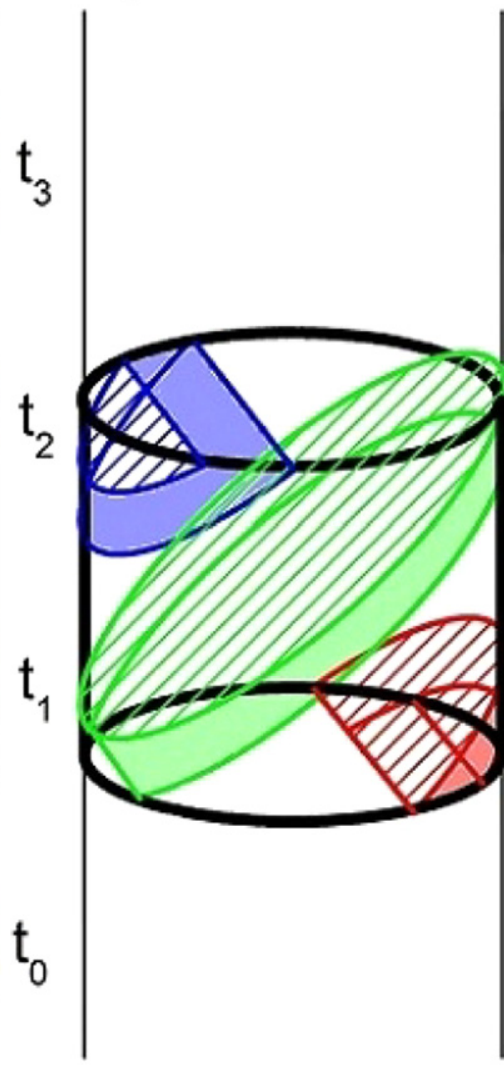

c)

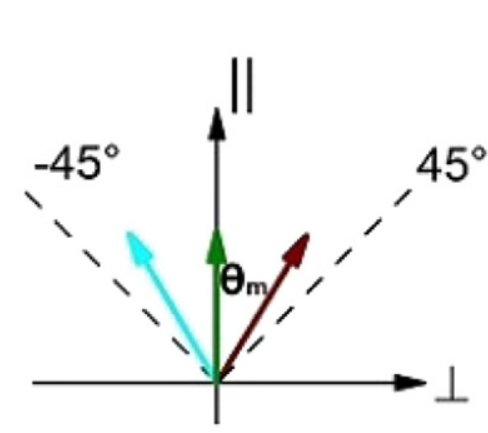

\section{LOS}

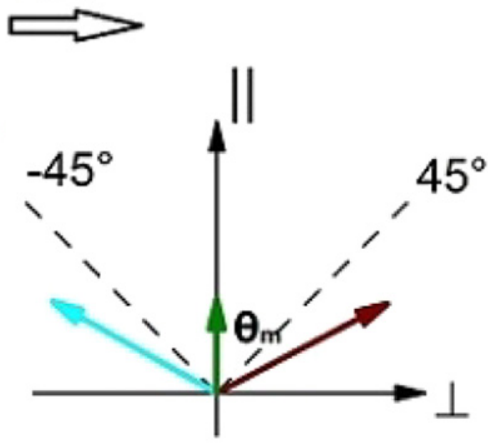

Figure 4. (a) Sketch of the interaction between the emission region and the disturbance in the comoving frame of the emission region, at different epochs. The emission region is pervaded by a helical magnetic field and a turbulent component (only the helical component is sketched). The disturbance is stationary in the observer's frame, but in the comoving frame of the emission region the disturbance is then moving up with Lorentz factor $\Gamma$. The part of the disturbance that first encounters the emission region (shaded ellipses, the "front") is the location of the injection of relativistic particles. The orange, red, green, and blue regions refer to the locations of the disturbance before the flare $\left(t_{0}\right)$, the rising phase $\left(t_{1}\right)$, peak $\left(t_{2}\right)$, and declining phase $\left(t_{3}\right)$, respectively. (b) The red, green, and blue shapes indicate the shape and location of the flaring region, corresponding to the disturbance at $t_{1} \sim t_{3}$, respectively, observed simultaneously, taking into account the LTTEs. (c) Sketch of the projection of the helical magnetic field onto the plane of sky in the comoving frame. The upper panel illustrates the quiescent state, the lower panel the active state. The cyan, dark green, and maroon arrows represent the left side, center, and the right side of the emission region shown in (a), corresponding to the color regions in Figure 1, respectively. $\|(\perp)$ denotes components parallel (perpendicular) to the bulk motion direction. The two dashed lines indicate $\pm 45^{\circ}$.
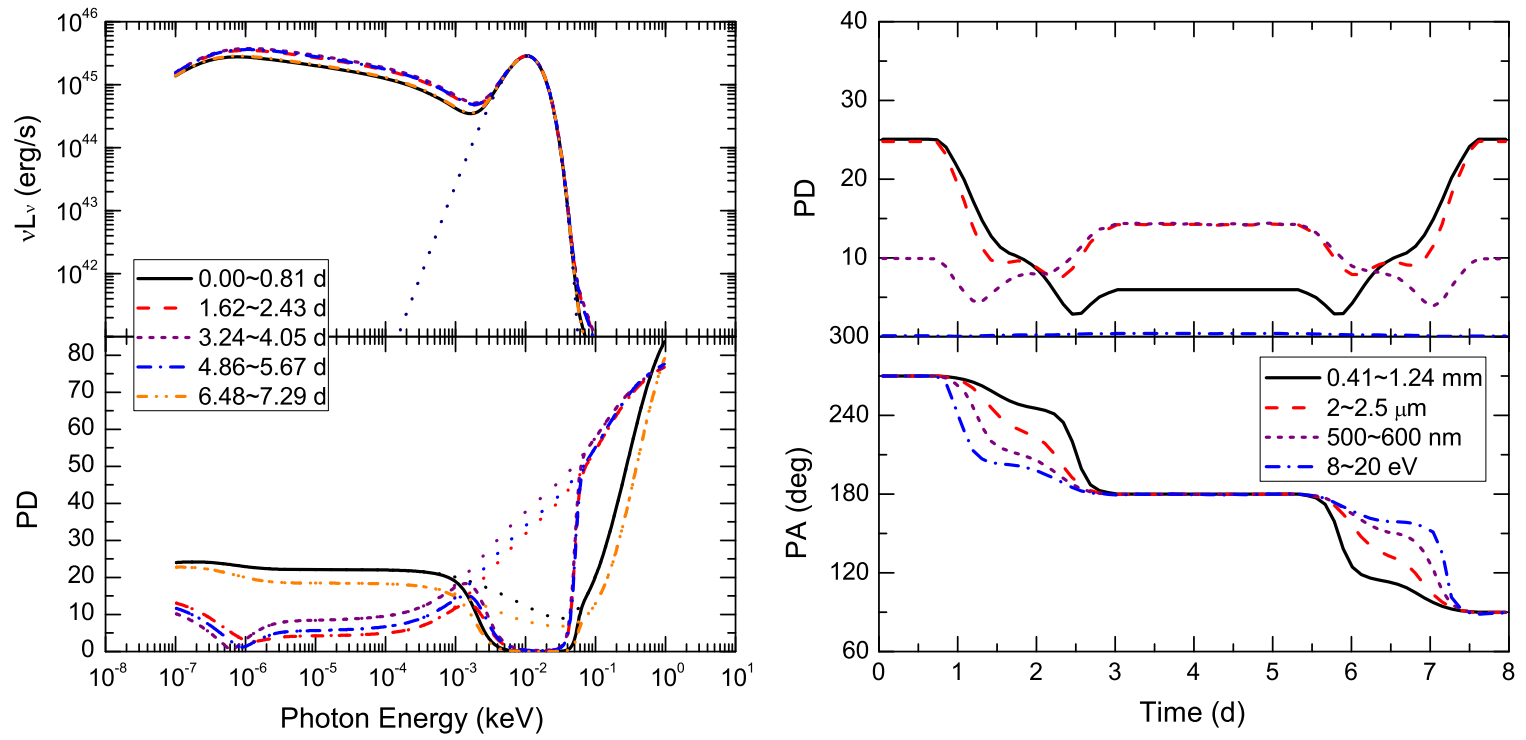

Figure 5. Case 2: a moderate change in magnetic field in Geometry II. Panels and line styles are as in Figure 2. 

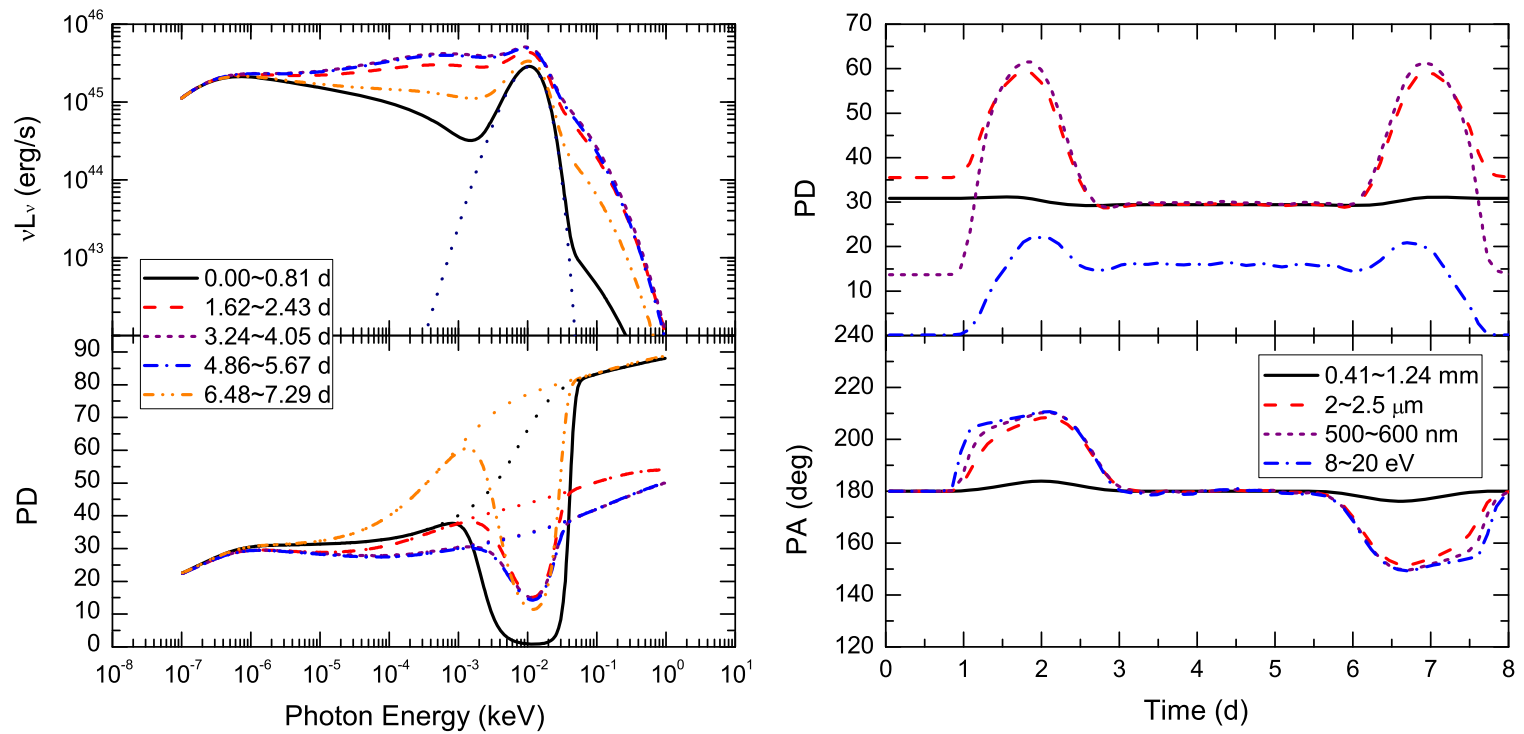

Figure 6. Case 3: shortened acceleration timescale in Geometry II. Panels and line styles are as in Figure 2.

etc. introduced by the different geometry. However, in Figure 5 we note that the flare amplitude is slightly increased, and thus the radio polarization signatures now behave similarly to the other bands. The major difference in this case is that in this scenario, the PD goes through two minima near zero with an elevated plateau in between. The PA exhibits a similar step-like pattern with a plateau at $180^{\circ}$, although, in total, it still completes a full $180^{\circ}$ swing. The reason for this is that unlike in the previous case, during the peak, the entire equal-photonarrival-time ellipse is maintained within the emission region for a finite amount of time, during which the polarization signatures remain unchanged.

\subsection{Acceleration Efficiency, $Z>2 R$}

We investigate one additional scenario where the shock leads to more efficient particle acceleration by instantaneously shortening the acceleration timescale at its location. Figure 6 shows the result. In this case, we choose the initial toroidal component to be slightly dominant with the application of Geometry II (we have shown one case with Geometry I in Paper I). Due to the more efficient acceleration, the electrons are accelerated to higher energy and the spectrum becomes harder (Figure 3 right). Therefore, the synchrotron component exhibits a considerable flare at higher energies. However, at lower energies, the electron spectrum stays almost unchanged. Since the magnetic field remains unchanged, the radio band shows no time dependency of either flux or polarization signatures.

At the beginning of the flare, the luminosity of the active region is drastically enhanced; as a result, the observed PD will mostly be attributed to the red region in Figure 4 (right). In this small region, the magnetic field is well ordered, and consequently we observe that PD can shoot up to over $60 \%$; meanwhile, PA quickly rotates to about $210^{\circ}$, which represent the $\theta_{B}$ in this region. Similar to the previous case, during the flare peak the entire emission region becomes axisymmetric, therefore PA rotates back and both PD and PA exhibit a step phase. After the peak, PD and PA revert back to the initial state in time-symmetric patterns.

\subsection{B-field Compression, Localized Shock}

We present one case for the combined geometric effect in Figure 7. This time we apply a moderate change in the magnetic orientation and strength during the presence of the shock with the same volume of the emission region as in Geometry I $\left(R: Z=1: \frac{4}{3}\right)$, but now the shock will not occupy the whole layer of the emission region, only the center of it, so that the region that will be affected by the shock has $A: Z=\frac{2}{3}: 3$ (hereafter, Geometry III). The current situation is equivalent to a Geometry II case but surrounded by a large quiescent region. Hence, the positive $q$ in the shock region will not be able to dominate over the negative $q$ in the quiescent region. Consequently, we find a behavior similar to Case 3 where the PA rotates back to $90^{\circ}$ and exhibits a step phase. The difference is that the PD slightly decreases during the flare peak, since the shock will give a boost to the toroidal contribution to the polarization, diminishing part of the initial poloidal contribution.

\section{APPLICATION TO 3C279}

Based on the insights gathered in the above study and Paper I, we now present the first simultaneous fitting of snapshot SEDs, multifrequency light curves, and time-dependent polarization signatures of a blazar using the example of a flare of 3C279. The FSRQ 3C279, located at a moderate redshift of $z=0.536$, is one of the most well-observed members of the blazar class. It caught the attention of the high-energy astrophysics community due to its very bright gamma-ray flaring at the beginning of the Compton Gamma-ray Observatory mission in the early 1990s, and since then has been the target of many dedicated multi-wavelength observing campaigns (Maraschi et al. 1994; Hartman et al. 1996; Wehrle et al. 1998; Abdo et al. 2010a). It is bright and variable over a large range of timescales, across the electromagnetic spectrum, from radio through gamma-rays, and is one of only three FSRQs detected in very-high-energy gamma-rays (i.e., photon energies of $E>100 \mathrm{GeV}$ ) by the MAGIC telescope (Albert et al. 2008). 

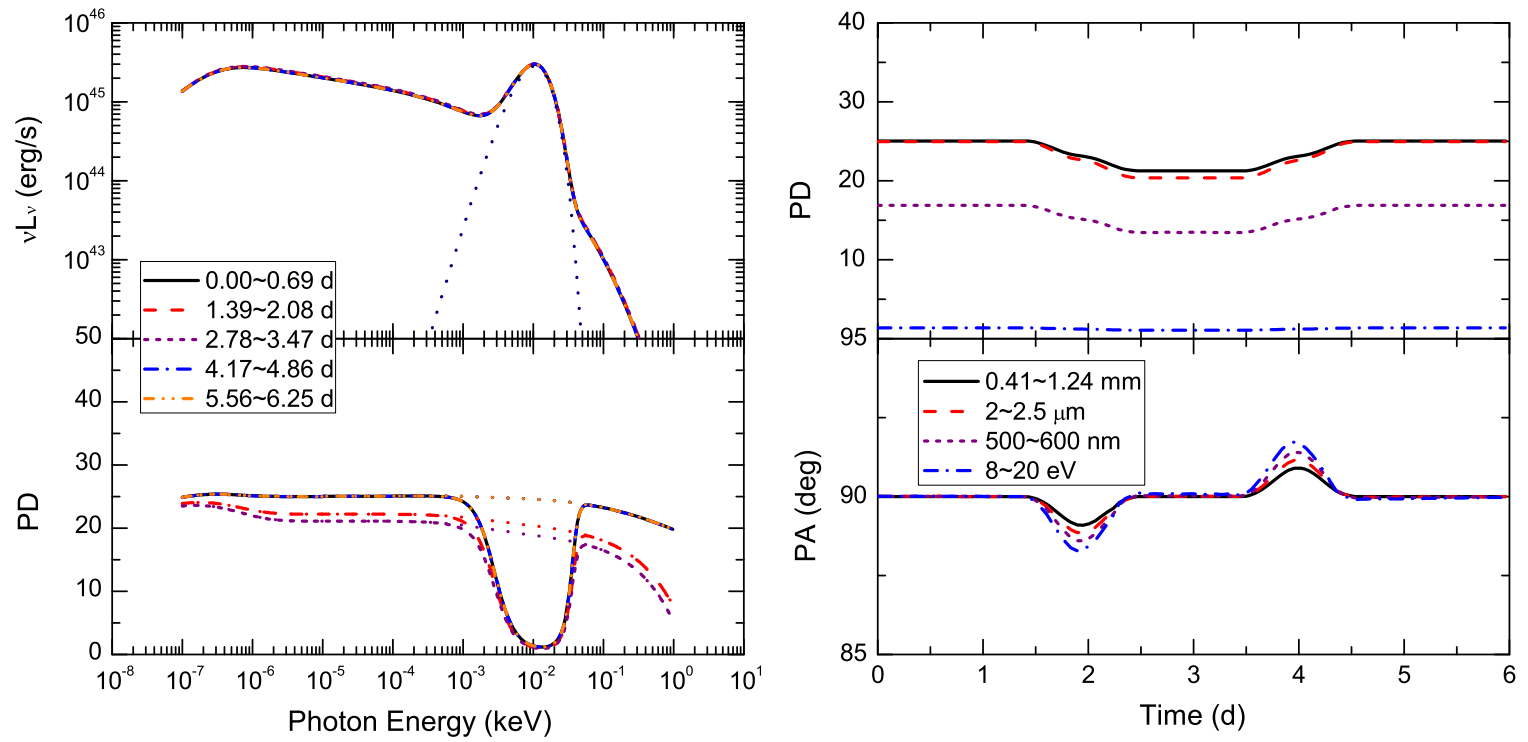

Figure 7. Case 4: moderate change of the magnetic field with Geometry III. Panels and line styles are as in Figure 2.

During an active (i.e., high-flux) phase from 2008 November to 2009 March, 3C279 exhibited multi-wavelength flaring activity with a period of optical polarization variations lasting 20 days (Abdo et al. 2010a). The multi-wavelength light curve data are displayed in Figure 8. They suggest that this flaring episode is actually composed of two sequential flares. The first flare dominates around MJD 54880, where a sudden doubling in gamma-ray flux is accompanied by a relatively small infrared-to-optical flare and rather erratic changes of the PD and PA. The second flare, which dominates the last $\sim 15$ days, constitutes a correlated flare of the infraredto-optical and gamma-ray emissions, during which the PD drops to nearly zero and then recovers to $\sim 20 \%$, accompanied by a $\sim 180^{\circ}$ rotation of the PA. This was the first time that such a clear correlation between optical/gamma-ray flaring and a PA rotation was observed. Based on the apparently time-symmetric profile of the second flare, we suggest that it actually lasted $\sim 20$ days, but is overwhelmed by the decaying phase of the first flare for the first $\sim 5$ days. Here, we present a consistent interpretation of the SED, multi-wavelength light curves, and time-dependent polarization features, for the second flare.

Table 2 lists the most relevant parameters in our simulation which yields the best fit to the data of $3 \mathrm{C} 279$, and the results are shown compared with the data in Figure 8. The lowfrequency component of the SED is dominated by synchrotron emission from nonthermal electrons, along with a weak thermal component from the central accretion disk around the black hole powering the relativistic jet. We assume a leptonic origin for the high-energy component of the SED, which is composed of an SSC contribution, dominating from X-rays to soft gamma-rays, and an EC contribution which dominates the emission in the Fermi $(\sim \mathrm{GeV})$ range. We also assume that there exists a turbulent magnetic field component which is initially uniform everywhere. We assume the disturbance to be associated with a magnetic energy dissipation process, e.g., magnetic reconnection, instead of a shock. It has been suggested that magnetic reconnection can dissipate a large fraction of the magnetic energy to produce a nonthermal power-law distribution of relativistic particles (Guo et al. 2014). A possible underlying physical picture is that on the trajectory of the emission region, it encounters a flat stationary region where the poloidal component of the helical magnetic field is in the opposite direction of that in the emission region. Therefore, the poloidal component will be dissipated during the presence of the disturbance due to magnetic reconnection, leading to particle acceleration (we use a simple injection to mimic this effect) and a locally stronger turbulent magnetic field component (Daughton et al. 2011; Guo et al. 2014).

The time dependence of the polarization signatures is similar to Case 1 of the previous section. Before interacting with the disturbance, the entire emission region contributes uniformly, and therefore, due to the axisymmetry of the underlying geometry and the mildly stronger poloidal component $\left(\theta_{B}=33^{\circ}\right)$ in the quiescent state, the polarization is dominated by the poloidal contribution. When the interaction with the disturbance starts (red in Figure 4), we assume that the poloidal component in this area decreases but the toroidal component remains nearly unaffected, and hence the quiescent state polarization will gradually be canceled out by the flaring toroidal contribution. As a result, the PD drops and the PA starts to rotate toward the dominant toroidal direction. As the disturbance moves forward, the flaring region becomes larger, resulting in increasing fluxes in both the synchrotron and Compton emissions. Toward the end of the interaction period, the flaring region (green in Figure 4) reaches its maximum size, leading to the observed flux maximum. Meanwhile, the magnetic field throughout the green flaring region in Figure 4 has approximately equal contributions from the near and far sides of the cylinder, mimicking the initial axisymmetry, except for a mildly stronger toroidal magnetic field contribution $\left(\theta_{B}=62^{\circ}\right)$. This flaring toroidal contribution is just strong enough to dominate over the poloidal contribution in the quiescent state. Consequently, the PD drops to nearly zero while the PA reflects the dominant toroidal magnetic field direction. After this flux peak, the flaring region gradually becomes smaller and moves to the upper left (blue in Figure 4), and the light curves and PD recover to their initial states with approximately time-symmetric profiles. However, since the toroidal component on the far side of the cylinder is opposite to that on the near side, the PA instead completes a $\sim 180^{\circ}$ swing 
Photon energy (eV)
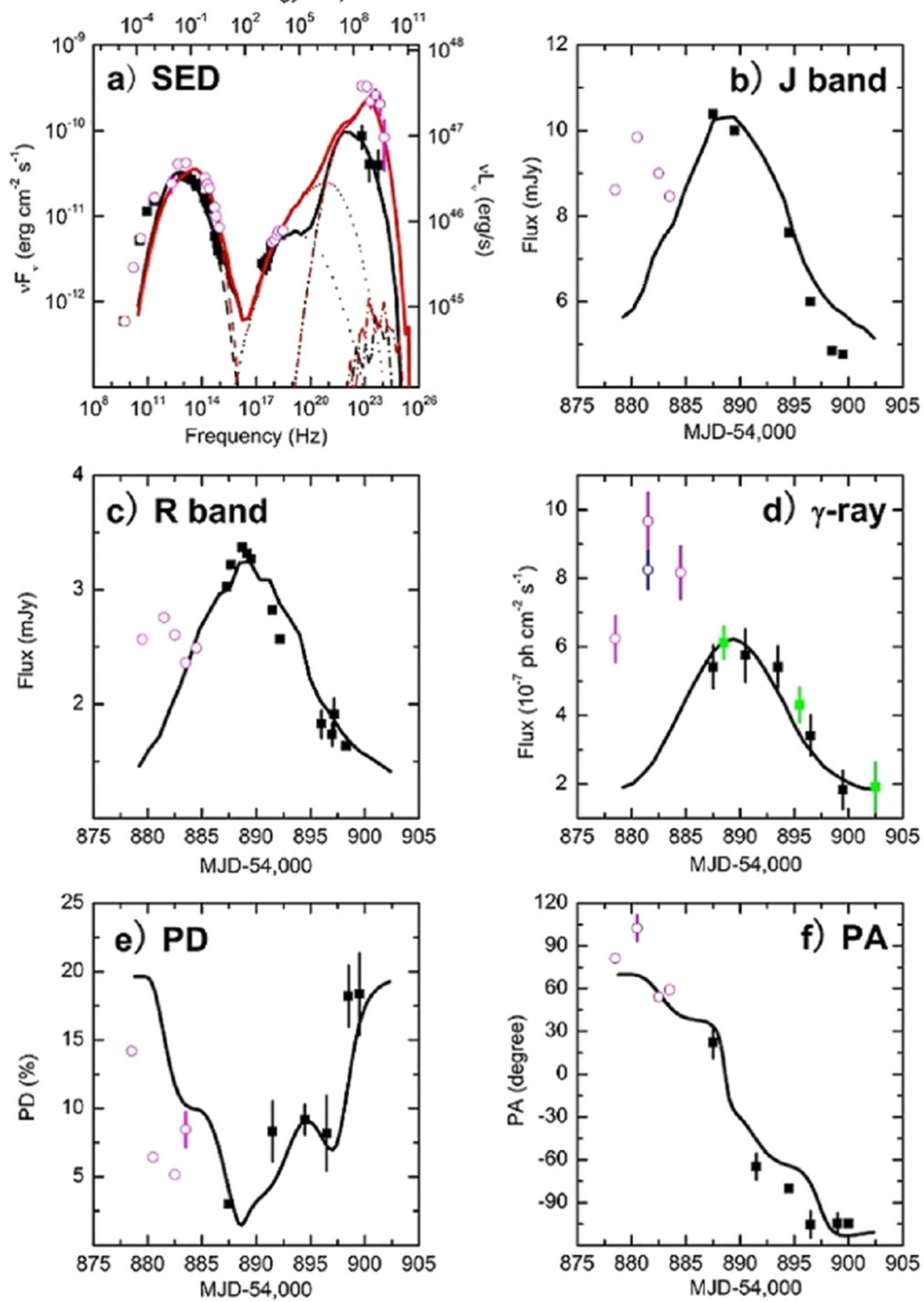

Figure 8. Data and model fits to multi-wavelength SEDs, light curves and polarization signatures during the second flare of 3C279 in 2009. Data are from Abdo et al. (2010a) and Hayashida et al. (2012). Hollow (filled) data points refer to the first (second) flare. (a) SED, black squares are from Period E in Hayashida et al. (2012; MJD 54897-54900), corresponding to the end of the second flare. The black curve is the model SED from the simulation at the same period; the red curve is the simulated SED at the peak of the flare. Hollow magenta circles are from Period D (MJD 54880-54885), corresponding to the end of the first flare. (b) and (c) J- and Rband flux: black squares are from the period of the second flare, hollow magenta circles are from the first flare; the black curves are the simulated light curves. (d) Gamma-ray photon flux light curve: black squares and hollow magenta circles are three day averaged data from the second and first flares, respectively, while green squares and hollow blue circles are the seven day averaged data, respectively. (e) and (f) PD and PA vs. time: black squares and hollow magenta circles are from the second and first flares, respectively; curves are the simulated polarization signatures.

to a direction that is indistinguishable from its initial position due to the $180^{\circ}$ ambiguity of the PA.

\section{DISCUSSIONS}

Polarization signatures are known to be highly variable, and $\geqslant 180^{\circ}$ polarization angle swings are frequently observed (e.g.,
Larionov et al. 2013; Morozova et al. 2014). Generally, the observed $\geqslant 180^{\circ} \mathrm{PA}$ swings are accompanied by one or several sequential apparently symmetric PD patterns which drop from an initial value to zero then revert back. In addition, both the PD and PA patterns appear to be smooth. Several mechanisms have been proposed to interpret the PA rotations, such as an emission region moving along a curved trajectory or a bending 
Table 2

Summary of Parameters

\begin{tabular}{|c|c|c|}
\hline$\overline{\text { Parameters }}$ & \multicolumn{2}{|c|}{ General Properties } \\
\hline Bulk Lorentz factor $\Gamma$ & \multicolumn{2}{|c|}{20} \\
\hline Length of the emission region $Z\left(10^{17} \mathrm{~cm}\right)$ & \multicolumn{2}{|c|}{4.8} \\
\hline Radius of the emission region $R\left(10^{17} \mathrm{~cm}\right)$ & \multicolumn{2}{|c|}{2.4} \\
\hline Length of the disturbance $L\left(10^{17} \mathrm{~cm}\right)$ & \multicolumn{2}{|c|}{1.28} \\
\hline Orientation of LOS $\theta_{\mathrm{obs}}\left({ }^{\circ}\right)$ & \multicolumn{2}{|c|}{90} \\
\hline Electron acceleration timescale $t_{\mathrm{acc}}(Z / c)$ & \multicolumn{2}{|c|}{0.36} \\
\hline Electron escape timescale $t_{\mathrm{esc}}(Z / c)$ & \multicolumn{2}{|c|}{0.062} \\
\hline Electron background temperature $T\left(m_{e} c^{2} / k_{B}\right)$ & \multicolumn{2}{|c|}{100} \\
\hline Parameters & Quiescent & Active \\
\hline Total magnetic field strength $B\left(10^{-2} G\right)$ & 7.2 & 5.4 \\
\hline Helical magnetic strength, $B_{H}\left(10^{-2} G\right)$ & 4.8 & 2.6 \\
\hline Helical pitch angle $\theta_{B}\left(^{\circ}\right)$ & 33 & 62 \\
\hline Initial electron density $n_{e}\left(\mathrm{~cm}^{-3}\right)$ & 5.5 & $\cdots$ \\
\hline Injected electron minimum gamma $\gamma_{\min , \text { inj }}$ & $\cdots$ & 2000 \\
\hline Injected electron maximum gamma $\gamma_{\max \text {,inj }}$ & $\ldots$ & 4000 \\
\hline Injected electron power-law index $p_{\text {inj }}$ & $\cdots$ & 4 \\
\hline Injection rate $Q_{\text {inj }}\left(10^{43} \mathrm{erg}^{*} \mathrm{~s}^{-1}\right)$ & $\cdots$ & 5 \\
\hline
\end{tabular}

Notes. The parameters are defined in the same way as in Section 3, except for a few differences. In this fitting, we assume the radius of the disturbance is equal to that of the emission region, so that $A=R$. As we mentioned in Section 3, the initial electron distribution will evolve to an equilibrium according to the Fokker-Planck equation before interacting with the disturbance, and therefore we only list the initial electron density $n_{e}$, as the spectrum will be determined by the Fokker-Planck equation. We assume that the escaped electrons will be balanced by the electrons picked up from the thermal background with a temperature $T$. The magnetic field has two components, a helical component $B_{H}$ along with turbulence; the total magnetic field strength will be $B$. The disturbance will change the layers in the emission region at its location into an active state. In such a situation, the magnetic field energy will be dissipated so that both the total strength and the helical strength will decrease and the pitch angle will alter. The dissipated energy will become the energy resource for particle acceleration, which we handle by an injection at the front of the disturbance with an energy injection rate $Q_{\text {inj }}$ into the emission region with minimum and maximum Lorentz factors $\gamma_{\text {min,inj }}$ and $\gamma_{\text {max,inj }}$, and power-law index $p_{\text {inj. }}$. Both the initial and injected electrons will evolve according to the Fokker-Planck equation

jet, or streamlines following helical magnetic field lines, or stochastic activation of individual zones in a turbulent jet (Abdo et al. 2010a; Marscher et al. 2010; Marscher 2014). While they may have their own virtue in understanding polarization variations, none of these models has so far been able to explain spectral variability properties, symmetric light curve profiles, and correlated symmetric polarization variability features in one coherent model. On the other hand, the LTTEs coupled with an axisymmetric emission region in our model naturally explain the apparently time-symmetric features of multi-wavelength light curves and polarization variations and their intrinsic correlations, which also appears to be the simplest model with the smallest number of fine-tunable parameters.

Based on our model, the continuous $180^{\circ}$ PA swings will impose some serious constraints on the physical background of the emission region, namely, the pitch angles of the quiescent and the active states will be on each side of a critical point $\theta_{c}$, and there will be a moderate flare strength, a moderate change of the pitch angle of the helical magnetic field, and a geometry of the emission region where $Z \gtrsim R$. If the pitch angles of the quiescent state and the active state are both within the range of 0 to $\theta_{c}$ or $\theta_{c}$ to $90^{\circ}$, where in our case $\theta_{c} \sim 55^{\circ}$, then the projected magnetic field will always provide the total Stokes parameters in the form of $1,-|q|, u$ or $1,|q|, u$, respectively, resulting in the PA fluctuating around $270^{\circ}$ or $180^{\circ}$, respectively. Additionally, if the flare amplitude or the pitch angle change is too weak, then throughout the flaring activity the total polarization signatures will be dominated by the quiescent state, and therefore PA will not complete a $180^{\circ}$ swing. Meanwhile, if on the other hand they are too strong, then the active region will dominate the polarization signatures for a much longer time, giving rise to a step-like feature as shown in Paper I. Note, however, that this feature is different from the "step-phase" feature introduced by a $Z>2 R$ geometry (Case 2) because the step-like feature caused by strong variations can only reach $180^{\circ}$ at the very peak of the flare, where the $u$ component of the Stokes parameters can be fully canceled out. On the other hand, the "step phase" due to the geometry can be diagnosed in the observation as completely flat (Ikejiri et al. 2011).

In addition to the above constraints, a simultaneous fitting of all spectral, light curve, and polarization properties can exclude a wide range of possible scenarios. Therefore, our fitting results place unprecedented constraints, most of which do not depend on the details of the model. Since in a leptonic model interpretation, the gamma-rays from 3C279 are produced by the EC process, an excess of either external photon field or nonthermal electrons is necessary to produce a gamma-ray flare. However, the former fails to produce the data, since such excess is unlikely to generate a correlated, time-symmetric multi-wavelength flare, but instead may lead to an anti-correlated behavior in the synchrotron emission due to excess radiative cooling. Also, the infrared-to-optical flare amplitude is smaller than that of the gamma-ray flare, and the X-ray emission, which represents the low-energy end of the SSC emission, shows almost no variability. This implies that the total magnetic field strength has to decrease during the flare. We suggest that the dissipated magnetic energy can be converted into nonthermal particle energy through magnetic reconnection. Most importantly, as we have discussed above, a smooth PA rotation and the nearly zero PD at the flare peak (green in Figure 4) imply that the toroidal magnetic field component should be mildly stronger than the poloidal one in the active state. However, since the excess of nonthermal electrons and the amount of the magnetic field alteration, which are linked by the reconnection process, have been well constrained, the poloidal and toroidal B-field components should remain comparable throughout the process. Moreover, the relative percentage of the turbulent magnetic field contribution increases in the active state. Nevertheless, we note that just like most multiwavelength models of blazar emission, our model underestimates the radio flux, and also overestimates the PD there. This is because the radio emission is known to be produced on larger scales at which the magnetic field appears more disordered. In addition, the environment in which our emission region is located is still opaque to radio emission.

Models of relativistic shocks propagating through the jet have been widely used to explain blazar flaring activities (Marscher \& Gear 1985; Spada et al. 2001; Joshi \& 
Böttcher 2007; Böttcher \& Dermer 2010). Such models naturally provide excess nonthermal electrons. However, they are expected to compress the magnetic field, leading to a change in $\theta_{B}$ with an increase in the overall magnetic field strength. Consequently, shocks are unlikely to fit our constraints. Alternatively, the fitting constraints strongly favor a magnetic energy dissipation process during the flare. We find in our simulation that the dissipated magnetic energy during the disturbance is comparable to the necessary amount of particle energy increase required to generates the flare. Simulations of magnetic energy dissipation have demonstrated that the energy stored in magnetic shear can be efficiently converted into a power-law distribution of relativistic particles (Guo et al. 2014). This process will reduce the magnetic field component that is subject to dissipation and can therefore change the magnetic field pitch angle. Moreover, the magnetic field topology inside the dissipation zone is likely to become turbulent, and thus it will strengthen the turbulent magnetic field contribution (Daughton et al. 2011).

For the smaller fluctuations in the polarization signatures (generally PA varies by $<90^{\circ}$ ), although some apparently symmetric profiles can be noted, the general patterns appear very complicated (Jorstad et al. 2013; Covino et al. 2015). In addition, those fluctuations often happen during the quiescent states, with lower PD. This implies that some inhomogeneity and more complex geometry, or some turbulence, are required (Marscher 2014).

We suggest that if a step phase along with time-symmetric variability of the polarization features is observed, especially in the PA profile, as it will be less affected by a possible turbulent B-field component, this may serve as a powerful constraint on the size and the geometry of the emission region. Since the step phase will serve as a measure of the length of $Z-2 R$, while the two symmetric PA (PD) alterations will imply the length of $A$. In this way, the size and the geometry of the region that is affected by the disturbance can be constrained while the size of the entire emission region can then be estimated by the flux and polarization percentage.

We thank the anonymous referee for a careful review and very helpful suggestions to improve the paper. H.Z., F.G., and H.L. are supported by the LANL/LDRD program and by DoE/ Office of Fusion Energy Science through CMSO. X.C. acknowledges support by the Helmholtz Alliance for Astroparticle Physics HAP funded by the Initiative and Networking Fund of the Helmholtz Association. M.B. acknowledges support by the South African Research Chairs Initiative (SARChI) of the Department of Science and Technology and the National Research Foundation ${ }^{6}$ of South Africa. Simulations were conducted on LANL's Institutional Computing machines.

\section{REFERENCES}

Abdo, A. A., Ackermann, M., Ajello, M., et al. 2010a, Natur, 463, 919 Abdo, A. A., Ackermann, M., Agudo, I., et al. 2010b, ApJ, 721, 1425 Aharonian, F., Akhperjanian, A. G., Bazer-Bachi, A. R., et al. 2007, ApJL, 664, L71

Albert, J., Aliu, E., Anderhub, H., et al. 2008, Sci, 320, 1752

Aleksić, J., Ansoldi, S., Antonelli, L. A., et al. 2014, A\&A, 569, A46

Böttcher, M., \& Dermer, C. D. 2010, ApJ, 711, 445

Böttcher, M., Reimer, A., Sweeney, K., \& Prakash, A. 2013, ApJ, 768, 54

Chen, X., Chatterjee, R., Zhang, H., et al. 2014, MNRAS, 441, 2188

Chen, X., Fossati, G., Böttcher, M., \& Liang, E. 2012, MNRAS, 424, 789

Covino, S., Baglio, M. C., Foschini, L., et al. 2015, A\&A, submitted D’Ammando, F., Raiteri, C. M., Villata, M., et al. 2011, A\&A, 529, A145 Daughton, W., Roytershteyn, V., Karimabadi, H., et al. 2011, NatPh, 7, 539 Dermer, C. D., Schlickeiser, R., \& Mastichiadis, A. 1992, A\&A, 256, L27 Guo, F., Li, H., Daughton, W., \& Liu, Y. 2014, PhRvL, 113, 155005 Hartman, R. C., Webb, J. R., Marscher, A. P., et al. 1996, ApJ, 461, 698 Hayashida, M., Madejski, G. M., Nalewajko, K., et al. 2012, ApJ, 754, 114 Ikejiri, Y., Uemura, M., Sasada, M., et al. 2011, PASJ, 63, 639 Joshi, M., \& Böttcher, M. 2007, ApJ, 662, 884

Jorstad, S. G., Marscher, A. P., Smith, P. S., et al. 2013, ApJ, 773, 147 Larionov, V. M., Jorstad, S. G., Marscher, A. P., et al. 2013, ApJ, 768, 40 Lyutikov, M., Pariev, V. I., \& Gabuzda, D. C. 2005, MNRAS, 360, 869 Mannheim, K., \& Biermann, P. L. 1992, A\&A, 253, L21

Maraschi, L., Ghisellini, G., \& Celotti, A. 1992, ApJL, 397, L5

Maraschi, L., Grandi, P., Urry, C. M., et al. 1994, ApJL, 435, L91

Marscher, A. P., \& Gear, W. K. 1985, ApJ, 298, 114

Marscher, A. P., Jorstad, S. G., D’Arcangelo, F. D., et al. 2008, Natur, 452, 966 Marscher, A. P., Jorstad, S. G., Larionov, V. M., et al. 2010, ApJL, 710, L126 Marscher, A. P. 2014, ApJ, 780, 87

Morozova, D. A., Larionov, V. M., Troitsky, I. S., et al. 2014, AJ, 148, 42

Mücke, A., \& Protheroe, R. J. 2001, APh, 15, 121

Pushkarev, A. B., Gabuzda, D. C., Vetukhnovskaya, Y. N., \& Yakimov, V. E. 2005, MNRAS, 356, 859

Sikora, M., Begelman, M. C., \& Rees, M. J. 1994, ApJ, 421, 153

Spada, M., Ghisellini, G., Lazzati, D., \& Celotti, A. 2001, MNRAS, 325, 1559

Wehrle, A. E., Pian, E., Urry, C. M., et al. 1998, ApJ, 497, 178

Westfold, K. C. 1959, ApJ, 130, 241

Zhang, H., \& Böttcher, M. 2013, ApJ, 774, 18

Zhang, H., Chen, X., \& Böttcher, M. 2014, ApJ, 789, 66

\footnotetext{
6 Any opinion, finding, and conclusion or recommendation expressed in this material is that of the authors and the NRF does not accept any liability in this regard.
} 